\title{
Minimally invasive Ivor Lewis esophagectomy
}

\author{
Jon O. Wee, MD, ${ }^{a}$ and Christopher R. Morse, $\mathrm{MD}^{\mathrm{b}}$
}

Minimally invasive esophagectomy has the potential to reduce morbidity and mortality for a complex surgery. We outline a technique for performing a minimally invasive Ivor Lewis esophagectomy and review the current experience.

Since the early 1990s, laparoscopy has been used to provide minimally invasive surgery for upper gastrointestinal diseases. This has led to techniques to decrease the morbidity and mortality of a wide range of surgeries. The esophagectomy has traditionally required a laparotomy and sometimes a thoracotomy. Its access into 2 major body cavities was often associated with increased morbidity. Minimal access surgery with laparoscopy and thoracoscopy for esophageal resection has gained popularity in recent years as an alternative to the traditional approach. We outline a technique for performing a minimally invasive resection and review the current practice.

\section{TECHNIQUE}

\section{Patient Preparation and Abdominal Dissection}

A minimally invasive Ivor Lewis approach is the preferred method for esophageal resection for both benign and malignant lesions. Bowel preparation is not required because it often leads to patient dehydration, although a liquid diet the day before surgery is encouraged. Central lines are not necessary if large-bore peripheral access is acquired. Patients are placed supine, and an endoscopy is performed to evaluate the lesion and extent of resection. Although lower esophageal and gastroesophageal junction tumors are easily addressed through this approach, proximal tumors above $18 \mathrm{~cm}$ may be better approached through a minimally invasive 3-hole approach. A double-lumen endotracheal intubation is then performed. Initial laparoscopic ports are placed in the upper abdomen in a paramedian fashion at 5 sites. Once a liver retractor is placed, the abdomen is examined for peritoneal disease. Dissection is begun by taking down the gastrohepatic ligament and working to expose the right crus. The phrenoesophageal ligament is divided and the distal esophagus is mobilized, being careful to keep all of the surrounding tissue with the specimen.

From the Brigham and Women's Hospital, ${ }^{\mathrm{a}}$ Boston, Mass; and Massachusetts General Hospital, ${ }^{\mathrm{b}}$ Boston, Mass.

Disclosures: Authors have nothing to disclose with regard to commercial support.

Presented at the 3rd International Minimally Invasive Thoracic Surgery Summit, Boston, Massachusetts, October 7-8, 2011.

Received for publication Oct 24, 2011; revisions received Nov 27, 2011; accepted for publication Dec 28, 2011; available ahead of print Feb 1, 2012.

Address for reprints: Jon O. Wee, MD, Brigham and Women's Hospital, 75 Francis St, Boston, MA 02115 (E-mail: jwee@ partners.org).

J Thorac Cardiovasc Surg 2012;144:S60-2

0022-5223/\$36.00

Copyright (c) 2012 by The American Association for Thoracic Surgery

doi:10.1016/j.jtcvs.2011.12.051
The omentum is then divided distal to the gastroepiploic artery. A large omental skirt is helpful for later use. Dissection is carried up to the short gastric arteries where the dissection then moves close to the greater curvature. The left crus is then exposed, and the esophagus is further mobilized into the mediastinum. Further dissection is performed to mobilize the posterior stomach down to the pylorus. The left gastric artery is then dissected from the lesser curvature. Once the surrounding tissue is mobilized, a gastrointestinal anastomosis (GIA) stapler is placed at the base of the artery and divided. The gastric conduit is created using additional GIA staplers, starting at the incisura. The right gastric artery is often preserved. The stomach is divided up to the cardia with multiple fires of the endo-GIA stapler, keeping a $5-\mathrm{cm}$ margin along the greater curvature. Light tension superiorly and inferior on the stomach can extend the length of the conduit and allow the creation of a straight staple line.

Attention is then directed to placement of a laparoscopic feeding jejunostomy tube. Once this is complete, the conduit is reevaluated for ischemia. If stable, the distal end of the specimen is sutured to the proximal conduit.

\section{Thoracic Dissection}

The patient is placed in the left lateral decubitus position with right lung isolation. A slight forward tilt is helpful. Initial dissection is performed mobilizing the anterior esophagus away from the pericardium and bronchus. The subcarinal and periesophageal lymph nodes are kept with the specimen. The azygos vein is divided, and dissection is carried above this level to the thoracic inlet. The vagus nerves are divided at the level of the azygos vein. Posteriorly, the pleura is divided and the esophagus dissected away from the chest wall and aorta. The specimen is then delivered into the chest along with the gastric conduit. Orientation of the conduit is verified and the suture is divided, separating the specimen from the conduit.

Proximally, the esophagus is sharply divided. The posterior incision is increased by $3 \mathrm{~cm}$, and the specimen is removed. After gentle dilation of the esophageal opening, the anvil of an end-to-end anastomosis stapler is placed and sutured closed. A single-layer full-thickness running stitch is used to incorporate the mucosa around the anvil. Then a second purse string is placed outside this row to synch down on the anvil. The proximal end of the gastric conduit is opened along the staple line. The handle of the end-to-end anastomosis stapler is placed within the spike delivered along the greater curvature. If the tip of the conduit looks blue or ischemic, the conduit can be further delivered on the handle and the spike delivered out in a better 
perfused area. This is attached to the anvil, and the anastomosis is formed. A nasogastric tube is placed, and the open end of the gastric conduit is closed with a linear GIA stapler. The attached omentum is then loosely wrapped over the staple line and anastomosis and sutured in place. Copious irrigation is performed before closure with the drains left in place.

\section{Postoperative}

Postoperative management involved bowel rest with early resumption of tube feeds. An upper gastrointestinal study is performed in 1 week followed by resumption of a liquid diet.

\section{DISCUSSION}

Luketich and colleagues ${ }^{1}$ initially published a series of 222 minimally invasive esophagectomies performed with a 3-hole technique, thoracoscopy followed by laparoscopy, and cervical anastomosis. They reported reduced postoperative pain and pulmonary complications while comparing favorably with the best published open series with regard to morbidity, mortality, and oncologic outcomes. A recent meta-analysis ${ }^{2}$ further validates minimally invasive esophagectomy with a direct comparison with open techniques. The authors found no difference between open and minimally invasive esophagectomy regarding leak rate and 30day mortality. However, intraoperative blood loss, intensive care unit (ICU) stay, hospital stay, and total morbidity were lower in patients undergoing minimally invasive esophagectomy. A subanalysis revealed that respiratory complications were lower in the minimally invasive group. In terms of oncologic outcomes, there was no significant difference in the number of lymph nodes retrieved. As stated by the authors, their study has many limitations, but the data provided are informative.

An Ivor Lewis technique is favored by many because it allows for improved visualization of mediastinal structures, decreased frequency of recurrent laryngeal nerve injuries, a comprehensive thoracic lymph node harvest, and the creation of a tension-free anastomosis between the remnant esophagus and the gastric conduit. ${ }^{3}$ Perioperative outcomes and the development of complications after open transthoracic esophagectomy have been attributed to a variety factors, including age, pulmonary comorbidities, preoperative performance status, nutrition status, and neoadjuvant therapy. ${ }^{4}$ It was hypothesized that a minimally invasive Ivor Lewis esophagectomy may lead to fewer early perioperative complications, although early series were primarily composed of small case reports. ${ }^{5,6}$ Initial attempts used an abdominal hand port and a thoracoscopic handsewn anastomosis before moving to a totally minimally invasive approach.

Bizekis and colleagues ${ }^{7}$ reported one of the largest series to date of minimally invasive Ivor Lewis esophagectomies. Of these, the first 35 included a hybrid approach with a planned mini-thoracotomy. A completely laparoscopicthoracoscopic method was used in the last 15 patients in this series. The median length of stay was 9 days, with the completely minimally invasive group having a shorter hospitalization (7 vs 9 days). The anastomotic leak rate was $6 \%$, and all pneumonias $(10 \%)$ occurred in the hybrid minithoracotomy group.

Tapias and Morse ${ }^{8}$ reported an initial series of 40 minimally invasive Ivor Lewis esophagectomies with $63 \%$ of patients receiving neoadjuvant therapy. All patients underwent an R0 resection with a median ICU stay of 1 day and hospital stay of 7 days. There were no anastomotic leaks and no 30-day mortality. Postoperative complications included 8 patients $(21 \%)$ with atrial fibrillation and 2 patients $(5 \%)$ with chylothorax, 1 requiring ligation. In an abstract recently reported, Sihag and colleagues ${ }^{9}$ compared open and minimally invasive Ivor Lewis at a single institution. A total of 38 minimally invasive and 74 open Ivor Lewis esophagectomies were performed for esophageal carcinoma. No difference was found in the adequacy of oncologic resections and 60-day mortality. However, the rate of pulmonary complications was significantly lower in the minimally invasive group at $2.6 \%$ compared with the open group at $40.5 \%$. In addition, the length of ICU and hospital stay, intraoperative blood loss, and amount of intraoperative fluids were also significantly decreased with a minimally invasive Ivor Lewis esophagectomy, whereas operative times were not.

\section{CONCLUSIONS}

Available data indicate that a minimally invasive Ivor Lewis esophagectomy can be performed safely with reasonable operative times, markedly less morbidity and mortality, excellent anastomotic integrity, and consistent initial oncologic outcomes. Because pulmonary complications carry a poor prognosis, the ability to decrease and eliminate them is critical, which seems to be achieved by a minimally invasive Ivor Lewis esophagectomy in several studies. Although long-term oncologic outcomes still need to be evaluated, initial short-term results indicate that a minimally invasive Ivor Lewis esophagectomy is at least equivalent. Certainly, further prospective studies are needed to confirm these results, such as a large multicentered, randomized, controlled trial.

\section{References}

1. Luketich JD, Alvelo-Rivera M, Buenaventura PO, et al. Minimally invasive esoph agectomy: outcomes in 222 patients. Ann Surg. 2003;238:486-95.

2. Nagpal K, Ahmed K, Vats A, et al. Is minimally invasive surgery beneficial in the management of esophageal cancer? A meta-analysis. Surg Endosc. 2010;24: 1621-9.

3. Pennathur A, Zhang J, Chen H, Luketich JD. The "best operation" for esophageal cancer? Ann Thorac Surg. 2010;89:S2163-7.

4. Reynolds JV, Ravi N, Hollywood D, et al. Neoadjuvant chemoradiation may increase the risk of respiratory complications and sepsis after transthoracic esophagectomy. J Thorac Cardiovasc Surg. 2006;132:549-55. 
5. Watson DI, Davies N, Jamieson GG. Totally endoscopic Ivor Lewis esophagectomy. Surg Endosc. 1999;13:293-7.

6. Nguyen NT, Follette DM, Lemoine PH, et al. Minimally invasive Ivor Lewis esophagectomy. Ann Thorac Surg. 2001;72:593-6.

7. Bizekis C, Kent MS, Luketich JD, et al. Initial experience with minimally invasive Ivor Lewis esophagectomy. Ann Thorac Surg. 2006;82:402-7.
8. Tapias LF, Morse CR. A preliminary experience with minimally invasive Ivor Lewis esophagectomy. Dis Esophagus. 2011 Oct 3 [Epub ahead of print].

9. Sihag S, Wright CD, Wain JC, et al. Comparison of perioperative outcomes following open versus minimally invasive Ivor Lewis esophagectomy at a single, high-volume center. Abstract presented at: 25th European Association of Cardiothoracic Surgery Annual Meeting; May 1-11 2011; Lisbon, Portugal. 